The recent work of Siegel, Sparrow and Hallman [6] has just come to the attention of the author. These workers have considered this problem in the heat transfer context. They report values of the eigenfunctions and eigenvalues which are in excellent agreement with the more extensive data of the present work.

5. Acknowledgment. The work reported here was carried out under the auspices of the Engineering Research Section of the American Cyanamid Company, Stamford, Connecticut. Permission to publish these results is hereby acknowledged with thanks.

The author is also indebted to S. Katz and J. Longfield of American Cyanamid for many helpful discussions concerning this problem.

Central Research Division

American Cyanamid Company

Stamford, Connecticut p. 202.

1. S. KAtz, "Chemical reactions catalyzed on a tube wall," Chem. Eng. Sci. v. 10, 1959,

2. L. Graetz, "Uber die Warmeleitungs fahigkeit von Flussigkeiten," Ann. Physik, v. 18,1883 , p. 79.

3. G. M. Brown, "Heat or mass transfer in a fluid in laminar flow in a circular or flat conduit," A. I. Ch. E. J., v. 6, 1960, p. 179.

4. J. B. Sellars, M. Tribus, \& J. S. Klein, "Heat transfer to laminar flow in a round tube or flat conduit- the Graetz problem extended," Trans. A. S. M.E., v. 78, 1956, p. 441.

5. F. B. Hildebrand, Introduction to Numerical Analysis, McGraw-Hill Publishing Co., New York, 1956, p. 238 .

6. R. Siegel, E. M. Sparrow \& T. M. Hallman, "Steady laminar heat transfer in a circular tube with prescribed wall heat flux," Appl. Sci. Res., v. A7, 1958, p. 386.

\title{
Efficient Continued Fraction Approximations To Elementary Functions
}

\section{By Kurt Spielberg}

1. Introduction. This paper describes an application and extension of the work of H. J. Maehly [1] on the rational approximation of $\operatorname{arc} \tan x$, and of E. G. Kogbetliantz [2], who developed Maehly's procedure so as to be applicable to the computer programming of elementary transcendental functions.

It is to be shown here that certain modifications, such as the introduction of terms which are easily computed on specific computers, lead to considerable improvements. In particular, the application of the modified method to several elementary functions will be described and corresponding final results will be given. Some of these approximations have been used with great success to develop subroutines for the IBM 704 and 709 computers. Our experience indicates that the method of Maehly and Kogbetliantz, as modified below, is superior to other current numerical procedures.

2. The Modified Method of Maehly and Kogbetliantz. The basic idea made use of by H. J. Maehly in connection with $f(x)=\operatorname{arc} \tan x$ is to approximate the function $f(x)$ by a ratio of two Chebyshev sums of order $k$

Received in final revised form December 21, 1960. 


$$
\begin{aligned}
& f(x)=\sum_{r=0}^{k} a_{r} \cdot T_{r}(x) /\left[1+\sum_{s=1}^{k} b_{s} \cdot T_{s}(x)\right] \\
&+H(x) /\left[1+\sum_{s=1}^{k} b_{s} \cdot T_{s}(x)\right] \equiv f^{*}(x)+A
\end{aligned}
$$

where $H(x)=\sum_{j=0}^{\infty} A_{j}{ }^{\left({ }^{k}\right)} \cdot T_{2 k+1+j}$, and $A$ is the absolute error.

If the coefficients $b_{s}$ are small, which will normally be the case for $-1 \leqq x \leqq 1$ and reasonably rapid convergence of the power series for $f(x)$, then the denominator of the error term is close to one over the interval of approximation and $H(x)$ represents the absolute error $A$ with sufficient accuracy (compare [3]). The order $k$ is chosen so as to keep $A$ below the desired upper limit of accuracy. In order to evaluate the unknown coefficients $a_{r}$ and $b_{s}$, one must know the coefficients $c_{n}$ of the Chebyshev expansion of $f(x)$. A comparison of that expansion with (1) leads, after use of the identity

$$
2 T_{m}(x) \cdot T_{n}(x) \equiv T_{m+n}(x)+T_{m-n}(x)
$$

to a set of $2 k+1$ simultaneous linear equations in the $2 k+1$ coefficients $a_{r}$ and $b_{s}$. Additional equations can be established for the coefficients $A_{j}{ }^{(k)}$ in the error function $H(x)$. For even and odd functions the subscripts of (1) are changed as follows: even, $r \rightarrow 2 r, s \rightarrow 2 s, j \rightarrow 2 j, k \rightarrow 2 k+\frac{1}{2}$; odd, $r \rightarrow 2 r+1, s \rightarrow 2 s, j \rightarrow 2 j$, $k \rightarrow 2 k+1$.

When this scheme was applied in practice, several additional ideas suggested themselves. They can be listed briefly as follows:

a) Application of the method to functions that can be expressed as ratios of Chebyshev series, such as $\tan \left(\frac{1}{4} \pi x\right)$.

b) Use of different degree numerator and denominator polynomials in (1).

c) Consideration of unequal intervals for two complementary expansions, such as $\sin \alpha x$ and $\cos \beta x, \alpha+\beta=\pi / 2$.

d) Reduction of the relative error by means of a linear correction term in a neighborhood of $x=0$.

e) Reduction of the error term through introduction of a new parameter that does not lead to a full additional multiplication.

The first three points should become clear in the sequel and need little amplification. Point $d$ is usually of concern for odd functions $f(x)$, if it is desired to obtain accurate results for $g(x)=f(x) / x$ as $x \rightarrow 0$. Chebyshev methods applied to the function $f(x)$ produce an approximation $f^{*}(x)$ such that the absolute error $\left|f(x)-f^{*}(x)\right|$ is (approximately) minimized over an interval such as $-1 \leqq x \leqq 1$. The relative error of such an approximation, $\left|f^{*}-f\right| / f$, usually becomes intolerably large as $x$ and $f$ approach zero. The natural way to cope with this difficulty would be to apply the Chebyshev approximation method to $g(x)$ rather than $f(x)$. Then the relative error $\left|f^{*}-f\right| / f=\left|x \cdot g^{*}-x \cdot g\right| / x \cdot g=\left|g^{*}-g\right| / g$ is nearly minimized over the interval if $g(x)$ does not vary too much. This approach, however, has the drawback that the improvement in the neighborhood of zero is paid for with a decrease in accuracy in the remainder of the interval. We have found that computer subroutines can easily be written so as to use $f^{*}(x)$ in most of the interval and 
$f^{*}(x)+x \cdot C$ in an appropriately chosen neighborhood of zero. The choice of the correction term $C$ will be discussed further below.

The most important modification of the method, point $e$, arises if the special machine characteristics of digital computers are taken into account. The reduction of the error clearly depends on the introduction of the parameters $a_{i}$ and $b_{i}$. Each new parameter allows reduction of one more error term $A_{i}$ to zero, but also results in an increase of the number of multiplications (or divisions), $M$, by one. We can, however, achieve a compromise by restricting the last parameter to a set of values which may allow the correspondingly introduced multiplication to be performed in a manner particularly suited to the calculator in question. In the case of the IBM 704 the multiplication might be reduced to a shift (a "cheap" multiplication with a power of two), in the case of the IBM 709 to a variable length multiplication requiring little time. Among the permissible values for the newly introduced parameter, that one is chosen which allows a maximum reduction of the dominant error term. In other words, one of the residuals in the system of linear equations for the $a_{i}$ and $b_{i}$ is not reduced to zero but only below a certain value determined by the desired accuracy.

As an example, we may consider as our newly introduced parameter the coefficient $a_{5}$ in

$$
f(x)=\left(a_{1} T_{1}+a_{3} T_{3}+a_{5} T_{5}\right)\left(1+b_{2} T_{2}\right)^{-1}=x \cdot\left(K_{1}+K_{2} \cdot x^{2}+\frac{K_{3}}{x^{2}+K_{4}}\right) .
$$

Evidently $K_{2}=8 a_{5} / b_{2}$. In accordance with the above discussion we restrict $K_{2}$ to the form $2^{n}$ ( $n \cdots$ any integer), so that $a_{5}$ becomes restricted to the set of values $b_{2} \cdot 2^{n}$. The integer $n$ is chosen so as to reduce the absolute error as far as possible. Numerical details will be given in Section 3.

The modified procedure of Maehly and Kogbetliantz can now be outlined formally as follows. Given a function $f(x)$, find an approximation $f^{*}(x)$

$$
f(x)=\frac{\sum_{i=0}^{\infty} c_{i} T_{i}(x)}{\sum_{i=0}^{\infty} d_{i} T_{i}(x)}, \quad f^{*}(x)=\frac{\sum_{i=0}^{l} \dot{a}_{i} T_{i}(x)}{1+\sum_{i=1}^{m} b_{i} T_{i}(x)} .
$$

To determine the coefficients $a_{i}$ and $b_{i}$, we consider the absolute error

$$
\begin{aligned}
& f(x)-f^{*}(x)= {\left[\sum_{i=0}^{\infty} d_{i} T_{i}\left(1+\sum_{i=1}^{m} b_{i} T_{i}\right)\right]^{-1} } \\
& \cdot\left[\left(1+\sum_{i=1}^{m} b_{i} T_{i}\right) \sum_{i=0}^{\infty} c_{i} T_{i}-\sum_{i=0}^{l} a_{i} T_{i} \cdot \sum_{i=0}^{\infty} d_{i} T_{i}\right] \\
&=\left[\sum_{i=0}^{\infty} d_{i} T_{i}\left(1+\sum_{i=1}^{m} b_{i} T_{i}\right)\right]^{-1} \cdot \sum_{i=0}^{\infty} R_{i} \cdot T_{i}(x)
\end{aligned}
$$

The coefficients $R_{i}$ can be viewed as the residuals, usually rapidly diminishing in magnitude as $i$ increases, of an infinite number of linear equations in the $s$ unknowns $x_{i}: b_{1}, b_{2} \cdots b_{m}, a_{0}, a_{1} \cdots a_{l}$. 


$$
R_{i}=\sum_{j=1}^{s} e_{i j} \cdot x_{j}+f_{i}, \quad i=0,1,2,3, \cdots, s-1, \cdots .
$$

The $e_{i j}$ and $f_{i}$ are simple sums formed with the coefficients of the given function $f(x), c_{i}$ and $d_{i}$. For instance, the important special case of a polynomial $f(x)$ gives rise to the following residuals:

$$
\begin{gathered}
R_{0}=c_{0} b_{0}+\frac{1}{2} \sum_{n=1}^{m} b_{n} c_{n}-a_{0}, \quad b_{0}=1 \\
1 \leqq i \leqq m: \quad R_{i}=c_{i}+\frac{1}{2} c_{0} b_{i}+\frac{1}{2} \sum_{n=1}^{m} b_{n} \cdot\left(c_{n+i}+c_{|n-i|}\right)-a_{i} \\
m<i: \quad R_{i}=\frac{1}{2} \sum_{n=0}^{m} b_{n} \cdot\left(c_{n+i}+c_{i-n}\right)-a_{i}
\end{gathered}
$$

For $i>l$, the term $-a_{i}$ is omitted above.

Usually one sets the first $s$ residuals equal to zero so that the final error is determined by the absolute sum of the remaining residuals. Instead of this we endeavor to reduce the first $s+1$ residuals below a desired bound $\delta$, by introducing the additional parameter $a_{l+1}$

$$
\begin{aligned}
R_{i} & =w_{i} \cdot \delta, & i=0,1, \cdots, s-1, s \\
x_{s+1}=a_{l+1} & =k \cdot x_{\nu}, & 1 \leqq \nu \leqq s .
\end{aligned}
$$

The $w_{i}$ are weightfactors which can be chosen arbitrarily, usually as 0 for $i<s$ and as 1 for $i=s$. The choice of $x_{\nu}$ and $k$ depends on the transformation from the rational approximation to the continued fraction. In the example given above $x_{\nu}$ is equal to $b_{2}$ and $k$ is chosen to be of the form $2^{n}$.

The residuals $R_{i}$ clearly become linear combinations of $s+1$ variables $x_{i}$. In view of (8), however, they can be expressed in terms of the first $s$ variables and $k$.

$$
R_{i}=w_{i} \cdot \delta=\sum_{j=1}^{\dot{C}} e_{i j} \cdot x_{j}+e_{i, s+1} \cdot k \cdot x_{\nu}+f_{i}, \quad i=0,1,2, \cdots, s-1, \quad 1 \leqq \nu \leqq s .
$$

These equations can now be solved for the $x_{j}$ in terms of $k$. As a consequence, one can determine the residual $R_{s}$ as a function of $k$

$$
x_{i}=x_{i}(k), \quad i=1,2, \cdots, s, \quad R_{s}=w_{s} \cdot \delta=f(k) .
$$

Finally one chooses among the manifold of permissible $k$, namely of those $k$ which permit the replacement of a multiplication by a more favorable operation, that value which minimizes $R_{s}$. It is usually possible to reduce $R_{s}$ so substantially that the leading term of the absolute error becomes $R_{s+1}$. Except for a bounded factor stemming from the denominator in (5), the final absolute error is given by

$$
A \cong \delta \cdot \sum_{i=0}^{\infty}\left|w_{i} \cdot T_{i}\right|+\sum_{i=s+1}^{\infty}\left|R_{i} \cdot T_{i}\right| \approx \sum_{i=s+1}^{\infty}\left|R_{i}\right|
$$

It is perhaps of interest to point out that, when applied numerically, this procedure 
usually produced values of $k$ which did not only reduce $R_{z}$ but also decreased $R_{s+1}$ in magnitude.

We finally turn our attention again to the correction term discussed in point $d$. Inspection of (11) indicates that in a sufficiently small neighborhood of $x=0$ one can approximate $A$ by the leading term $R_{s+1} \cdot T_{s+1} \cong R_{s+1} \cdot$ const $\cdot x$. The size of the interval about zero will primarily depend on the relative magnitude of the two lowest degree terms in the replaced Chebyshev polynomial. For instance, $\left|R_{9} \cdot T_{9}\right|=\left|R_{9} \cdot\left(256 x^{9}-576 x^{7}+432 x^{5}-120 x^{3}+9 x\right)\right|$ can be replaced by $\left|9 \cdot R_{9} \cdot x\right|$ for $|x| \ll 3 / \sqrt{120}$, or for a neighborhood of zero in which $120 \cdot R_{9} \cdot x^{2}<w_{9} \cdot \delta$. (Of course, $R_{11} \cdot T_{11}$ must also be below the tolerable error limit.) From a practical standpoint, it is simplest to compute the coefficient of the linear error term as $\lim _{x \rightarrow 0}\left[f(x) / x-f^{*}(x) / x\right]$.

3. Applications and Results. The method outlined above was applied to produce rational approximations for the development of optimal elementary function subroutines for the IBM 704 and 709 computers. The resulting routines were tested carefully and have been found to give, within the limits of roundoff error, results of the predicted accuracy.

a) Sine Approximation, 8-Digit Accuracy.

$$
\begin{aligned}
& \sin ^{*} \alpha x=2\left(a_{1} T_{1}+a_{3} T_{3}+a_{5} T_{5}\right)\left(1+b_{2} T_{2}\right)^{-1} \cong z\left(K_{1}+\frac{1}{4} z^{2}+\frac{K_{8}}{z^{2}+K_{4}}\right) \\
& \alpha=.3, \quad z=.3 x, \quad-.3 \leqq z \leqq .3
\end{aligned}
$$

In this case (10) becomes

$$
R_{3} \cong 10^{-9} \cdot\left[-.28029+10^{-3} \cdot .38076\left( \pm 2^{n-2} \cdot \alpha^{3}+10^{-3} \cdot .55934\right)^{-1}\right] .
$$

The minimum of $R_{3}$ is reached for $n=-3$. The correspondingly attained improvement becomes apparent if one compares $\left.R_{3}\right)_{-3}$ with $\left.R_{3}\right)_{-\infty}$, the error without correction term.

$$
\left.\left.R_{3}\right)_{n=-3} \cong .5 \times 10^{-10}, \quad R_{3}\right)_{n=-\infty} \cong .4 \times 10^{-9}
$$

In the sequel we shall give the results of our computations as they were calculated, that is to more places than is usually warranted by the accuracy.

$$
\begin{aligned}
& a_{1}=\quad .14838520812231 \quad K_{1}=-10^{2} \times .1984592426192 \\
& a_{3}=-10^{-3} .4926995891193 \quad K_{3}=10^{4} \times .1042926708144 \\
& a_{5}=\quad 1^{-6} .3791169631734=2^{n-3} \cdot \alpha^{3} b_{2}, \quad K_{4}=\quad 10^{2} \times .5003024548541 \\
& b_{2}=10^{-3} .8986476164110 \\
& \alpha=.3, \quad n=-3, \quad A \text { (absolute error) } \cong 10^{-10} \times .55, R \text { (relative error) } \\
& \cong 14 \mathrm{~A} / .2955 \cong 10^{-8} \times .26
\end{aligned}
$$

Check: 1$) \lim \left(\sin ^{*} z\right) / z=.99999999899$ as $z \rightarrow 0$

2) $\sin ^{*}(.15)=.149438132475 \cdots, \sin (.15)=.14943813 \cdots$ 
b) Cosine Approximation, 8-Digit Accuracy.

$$
\begin{aligned}
& \cos ^{*} 1.3 x=2\left(a_{0} T_{0}+a_{2} T_{2}+a_{4} T_{4}+a_{6} T_{6}\right)\left(1+b_{2} T_{2}+b_{4} T_{4}\right)^{-1} \\
& =K_{1}-2 z^{2}+K_{3}\left[z^{2}+K_{4}+K_{5}\left(z^{2}+K_{6}\right)^{-1}\right]^{-1} \\
& z=1.3 x, \quad-1.3 \leqq z \leqq 1.3 \\
& a_{0}=\quad .3081259625215 \quad K_{1}=10^{3} \times .33947714945237 \\
& \text { (13) } a_{2}=\quad-.1764668549891 \quad K_{3}=-10^{5} \times .29702036590243 \\
& a_{4}=10^{-2} \times .4937928852647 \quad K_{4}=10^{2} \times .57936139283225 \\
& a_{6}=-10^{-4} \times .3449621183611 \quad K_{5}=10^{4} \times .14546862657824 \\
& b_{2}=10^{-1} \times .2095116328571 \quad K_{6}=10^{2} \times .48789057406695 \\
& b_{4}=10^{-4} \times .8164783866535
\end{aligned}
$$

In this example equations (8) and (10) take the form:

$$
\begin{gathered}
a_{6}=2^{n-2} \cdot(1.3)^{2} \cdot b_{4}, \quad R_{5} \cong 10^{-8}\left(-.1490+2^{n-1} .3608\right)\left(.2862+2^{n-1} 1.667\right)^{-1} \\
\left.\left.R_{5}\right)_{n=-\infty} \cong-.52 \times 10^{-8}, \quad R_{5}\right)_{n=0} \cong .30 \times 10^{-9}
\end{gathered}
$$

$A$ (absolute error) $\cong .30 \times 10^{-9}, \quad R$ (relative error) $\cong .20 \times 10^{-8}$.

The actual computation of (13) involves the subtraction of two large numbers with a corresponding loss of accuracy. Hence a transformation to a more satisfactory continued fraction was performed

$$
\begin{array}{ll}
\cos ^{*} 1.3 x=H_{1}-2 z^{2}+\left(H_{3}+320 z^{2}\right)\left[z^{2}+H_{4}+H_{5}\left(z^{2}+H_{6}\right)^{-1}\right]^{-1} \\
H_{1}=10^{2} \times .19477149452366 & H_{5}=10^{4} \times .22874431956870 \\
H_{3}=-10^{4} \times .32763399516402 & H_{6}=10^{2} \times .24144894694287 \\
H_{4}=10^{2} \times .82580301995633 &
\end{array}
$$

Check: 1) $\cos ^{*}(0)=1.0000000005$

2) $\cos ^{*}(1)=.5403023025, \quad \cos (1)=.540302306 \cdots$

c) Tangent Approximation, 8-Digit Accuracy.

$$
\begin{aligned}
\tan \frac{1}{4} \pi x= & \left(a_{1} T_{1}+a_{3} T_{3}+a_{5} T_{5}\right)\left(1+b_{2} T_{2}\right)^{-1} \\
& =2\left[K_{1}+K_{2} z^{2}+K_{3}\left(z^{2}+K_{4}\right)^{-1}\right]+\underbrace{(.165) 10^{-6} z}_{\text {correction term }}
\end{aligned}
$$

$-\frac{1}{4} \pi \leqq z=\frac{1}{4} \pi x \leqq \frac{1}{4} \pi, \quad$ correction term added for $-.15 \leqq z \leqq .15$

$$
\begin{array}{lrlr}
a_{1}= & .8673936410 & K_{1}= & .18717826977 \\
a_{3}=-10^{-1} .1009694650 & K_{2}= & 10^{-2} .4150390625=2^{-7}(.10001)_{2} \\
a_{5}=-10^{-4} .3587664246 & K_{3}= & -10 & .20070072281 \\
b_{2}=-\quad .1427391684 & K_{4}=-10 & .24691855021 \\
A & \text { (absolute error) } \cong .842 \times 10^{-8}
\end{array}
$$


The relative error is reduced by the addition of the correction term.

Check: 1) $\tan ^{*} z / z=1.0000000001$ as $z \rightarrow 0$

2) $\tan ^{*}(.1)=.100334665, \quad \tan (.1)=.10033467 \ldots$

$3) \tan ^{*}(.7)=.8422883844, \quad \tan (.7)=.84228838 \cdots$

d) Cotangent Approximation, 8-Digit Accuracy.

$$
\begin{aligned}
\cot ^{*} \frac{1}{4} \pi x & =\left(a_{1} T_{1}+a_{3} T_{3}\right)\left(1+b_{2} T_{2}+b_{4} T_{4}\right)^{-1} \\
& =1 / z[K_{1}+K_{2} z^{2}+K_{3}\left(z^{2}+K_{4}\right)^{-1} \underbrace{\left..526 \times 10^{-7}\right]}_{\text {correction term }}
\end{aligned}
$$

$-\frac{1}{4} \pi \leqq z \leqq \frac{1}{4} \pi$, correction term added for $-.15 \leqq z \leqq .15$

$$
\begin{aligned}
& a_{1}=\quad .8636996360 \quad K_{1}=10 \times .34180166678 \\
& a_{3}=-10^{-1} \times .13359274436 \quad K_{2}=-\quad .1015625=-(.0001101)_{2} \\
& b_{2}=-\quad .15019244548 \quad K_{3}=\quad 10^{2} \times .252265398966 \\
& b_{4}=10^{-3} \times .53281462842 \quad K_{4}=-10^{2} \times .104327405083
\end{aligned}
$$

Check: 1) $z \cdot \cot ^{*} \mathrm{z}=1.0000000525-.526 \times 10^{-7}$ as $z \rightarrow 0$.

2) $\left[\cot ^{*} .1\right]^{-1}=.1003346678, \quad \tan .1=.10033467 \ldots$

3) $\left[\cot ^{*} .15\right]^{-1}=.151135221, \quad \tan .15=.15113522 \ldots$

e) Tangent Approximation, 10-Digit Accuracy.

$$
\begin{aligned}
& \tan * \frac{1}{4} \pi x=\left(a_{1} T_{1}+a_{3} T_{3}+a_{5} T_{5}\right)\left(1+b_{2} T_{2}+b_{4} T_{4}\right)^{-1} \\
& =z\left\{\left(K_{1}+K_{2} z^{2}\right)\left[z^{2}+K_{3}+K_{4}\left(z^{2}+K_{5}\right)^{-1}\right]^{-1}\right\} \\
& -\frac{1}{4} \pi \leqq z=\frac{1}{4} \pi x \leqq \frac{1}{4} \pi \\
& a_{1}=\quad .861300080500276 \quad K_{1}=-10^{1} \times .629934578714378 \\
& a_{3}=-10^{-1} \times .154784684131747 \quad K_{2}=\quad .0673828125 \\
& =(.1000101)_{2} \cdot 2^{-3} \\
& a_{5}=10^{-4} \times .23254407227 \quad K_{3}=-10^{2} \times .17890723138022 \\
& b_{2}=-\quad .155033946054144 \quad K_{4}=10^{3} \times .115116595703706 \\
& b_{4}=10^{-3} \times .878812556877435 \quad K_{5}=-10^{1} \times .993122665390157
\end{aligned}
$$

It was again found desirable to transform to an equivalent approximation with smaller coefficients:

$$
\begin{aligned}
& \tan ^{*} z=z\left\{\left(H_{1}+H_{2} z^{2}\right)\left[z^{2}+H_{3}+\left(H_{4}-16 z^{2}\right)\left(z^{2}+H_{5}\right)^{-1}\right]^{-1}\right\} \\
& H_{1}=K_{1}, \quad H_{2}=K_{2}, \quad H_{3}=-10^{1} \times .1890723138022 \\
& H_{4}=10^{2} \times .437830307587186 \quad H_{5}=K_{5} \\
& A \text { (absolute error) } \cong .7 \times 10^{-11}, \quad R \text { (relative error) } \cong .83 \times 10^{-10}
\end{aligned}
$$


Check: 1) $\lim (\tan *) / z=1.0000000000197$ as $z \rightarrow 0$.

2) $\tan * \frac{1}{4} \pi x=(.78539816352) .4 / \pi, \quad \frac{1}{4} \pi=.785398163 \cdots$

f) Sine Approximation, 10-Digit Accuracy.

$$
\begin{aligned}
\sin * \frac{1}{4} \pi x & =2\left(a_{1} T_{1}+a_{3} T_{3}+a_{5} T_{5}\right)\left(1+b_{2} T_{2}+b_{4} T_{4}\right)^{-1} \\
& =z\left\{K_{1}+K_{2}\left[z^{2}+K_{3}+K_{4}\left(z^{2}+K_{5}\right)^{-1}\right]^{-1}\right\} \\
& =z\left\{H_{1}+\left(H_{2}+6 z^{2}\right)\left[z^{2}+H_{3}+H_{4}\left(z^{2}+H_{5}\right)^{-1}\right]^{-1}\right\}
\end{aligned}
$$

$-\frac{1}{4} \pi \leqq z=\frac{1}{4} \pi x \leqq \frac{1}{4} \pi$

$a_{1}=\quad .364984470884912 \quad K_{1}=\quad 10^{1} \times .714830266086945$

$a_{3}=-10^{-2} \times .785959936053327 \quad K_{2}=-10^{3} \times .802850002448424$

(19)

$a_{5}=10^{-4} \times .304252059202251 \quad K_{3}=10^{2} \times .550726577370549$

$b_{2}=10^{-1} \times .10166051940260 \quad K_{4}=10^{2} \times .12558999435548$

$b_{4}=10^{-4} \times .21677076180 \quad K_{5}=10^{2} \times .166326525462696$

$H_{1}=10^{1} \times .114830266086945 \quad H_{4}=10^{4} \times .21114873690673$

$H_{2}=-10^{3} \times .377734420959983 \quad H_{5}=\quad .852713368584500$

$H_{3}=10^{2} \times .708525969147400$

$A$ (absolute error $) \cong .4356 \times 10^{-11}, \quad R$ (relative error $) \cong .863 \times 10^{-10}$

Check: 1) $\lim \sin ^{*} z / z=.999999999977$ as $z \rightarrow 0$

2) $\sin *(.5)=.4794255386, \quad \sin (.5)=.479425539 \cdots$

g) Cosine Approximation, 10-Digit Accuracy.

$$
\begin{aligned}
& \cos ^{*} \frac{1}{4} \pi x=2\left(a_{0} T_{0}+a_{2} T_{2}+a_{4} T_{4}+a_{6} T_{6}\right)\left(1+b_{2} T_{2}+b_{4} T_{4}\right)^{-1} \\
& =K_{1}-2 z^{2}+K_{2}\left[z^{2}+K_{3}+K_{4}\left(z^{2}+K_{5}\right)^{-1}\right]^{-1} \\
& =H_{1}-2 z^{2}+\left(H_{2}+338 z^{2}\right)\left[z^{2}+H_{3}+H_{4}\left(z^{2}+H_{5}\right)^{-1}\right]^{-1} \\
& -\frac{1}{4} \pi \leqq z=\frac{1}{4} \pi x \leqq \frac{1}{4} \pi \\
& a_{0}=\quad .425535314592886 \quad K_{1}=\quad 10^{3} \times .338416562920989 \\
& a_{2}=-10^{-1} \times .699507190410770 \quad K_{2}=-10^{5} \times .294738908526762 \\
& a_{4}=10^{-3} \times .684764823911432 \quad K_{3}=10^{2} \times .574514174244846 \\
& \text { (20) } \quad a_{6}=-10^{-5} \times .170461706567264 \quad K_{4}=10^{4} \times .146160003497481 \\
& b_{2}=10^{-2} \times .7666047537720 \quad K_{5}=10^{2} \times .488825769511137 \\
& b_{4}=10^{-4} \times .11053684400 \\
& H_{1}=\quad .416562920989 \quad H_{4}=10^{4} \times .422830564242266 \\
& H_{2}=10^{4} \times .63340598412301 \quad H_{5}=\quad .3933118492483 \\
& H_{3}=10^{3} \times .10594068252635 \\
& R(\text { relative error }) \cong .5 \times 10^{-11}
\end{aligned}
$$


h) Logarithm Approximation, 8-Digit Accuracy.

$$
\begin{aligned}
& \log _{2}{ }^{*} f=Z\left[K_{0}+K_{1} \cdot Z^{2}+K_{2}\left(K_{3}+Z^{2}\right)^{-1}\right]-\frac{1}{2}, \quad Z \equiv \frac{f-\frac{1}{2} \sqrt{2}}{f+\frac{1}{2} \sqrt{2}} \\
& \frac{1}{2} \leqq f<1 \\
& \text { (21) } K_{0}=10^{1} \times .186646762369 \\
& K_{2}=10^{1} \times .1354503944219 \\
& \left.K_{1}=\quad .1953125 \equiv .0011001\right)_{2} \quad K_{3}=10^{1} \times .132934939797 \\
& A \text { (absolute error }) \cong 2 \times 10^{-8}
\end{aligned}
$$

Several of the above approximations listed below have been incorporated in an Elementary Function Subroutine Package for the IBM computers 704 and 709.

$\begin{array}{llcc}\text { Equation } & \text { Machine } & \begin{array}{c}\text { Share Distribution } \\ \text { Number }\end{array} & \text { Name } \\ (12),(14) & 704,709 & 510,507 & \text { IB SIN1 } \\ (16) & 704,709 & 510,507 & \text { IB TAN1 } \\ (17) & 704,709 & 510,507 & \text { IB TAN2 } \\ (19),(20) & 704,709 & 571,590 & \text { IB SIN2 } \\ (21) & 709 & 665 & \text { IB LOG3 }\end{array}$

In conclusion, the author wishes to express his indebtedness to Dr. E. G. Kogbetliantz for his advice and guidance, and to Mr. F. S. Beckman, IBM, for the support of this project.

IBM Corporation, New York, and

College of the City of New York

1. H. J. MaEhly, Monthly Progress Report (unpublished), February 1956, Electronic Computer Project, The Institute for Advanced Study, Princeton.

2. E. G. Kogbetliantz, "Report No. 1 on 'Maehly's method, improved and applied to elementary functions' subroutines," April 1957, Service Bureau Corp., New York.

3. K. SPIELBERG, "The representation of power series in terms of polynomials, rational approximations, and continued fractions," submitted to J. Assoc. Comput. Mach.

4. C. Lanczos, Applied Analysis, Prentice-Hall, Inc., New York, 1956.

5. E. G. Kogbetriantz, Papers on Elementary Functions in IBM J. Res. \& Develop., v. 1, no. 2 ; v. 2 , no. 1 ; v. 2 , no. $3 ;$ v. 3 , no. $2 ; 1957-1959$.

6. E. G. KogBetuantz, "Generation of elementary functions" in A. Ralston \& H. S. Wilf, Mathematical Methods for Digital Computers, John Wiley \& Sons, Inc., New York, 1959.

7. Hans J. MaEhly, "Methods for fitting rational approximations," Part 1, J. Assoc. Comput. Mach., v. 7, 1960 , p. 150.

8. F. D. Murnaghan \& J. W. Wrench, Jr., "The determination of the Chebyshev approximating polynomial for a differentiable function," $M T A C, \mathrm{v} .13,1959, \mathrm{p} .185$.

9. P. WYNN, "The rational approximation of functions which are formally defined by a power series expansion," Math. Comp., v. 14, 1960, p. 147. 